\title{
Study of Sign Language Translation using Gesture Recognition
}

\author{
Neha Poddar ${ }^{1}$, Shrushti Rao $^{2}$, Shruti Sawant ${ }^{3}$, Vrushali Somavanshi ${ }^{4}$, Prof. Sumita Chandak ${ }^{5}$ \\ BE, IT Department, Atharva College of Engineering, Mumbai, India ${ }^{1,2,3,4}$ \\ Professor, ME, IT Department, Atharva College of Engineering, Mumbai, India ${ }^{5}$
}

\begin{abstract}
Communication is an integral part of human life. But for people who are mute \& hearing impaired, communication is a challenge. To understand them, one has to either learn their language i.e. sign language or finger language. The system proposed in this project aims at tackling this problem to some extent. In this paper, the motivation was to create an object tracking application to interact with the computer, and develop a virtual human computer interaction device. The motivation behind this system is two-fold. It has two modes of operation: Teach and Learn. The project uses a webcam to recognize the hand positions and sign made using contour recognition [3] and outputs the Sign Language in PC onto the gesture made. This will convert the gesture captured via webcam into audio output which will make normal people understand what exactly is being conveyed. Thus our project Sign Language to Speech Converter aims to convert the Sign Language into text and audio.
\end{abstract}

Keywords: Gesture recognition, image processing, Edge detection, Grey scale image

\section{INTRODUCTION}

The project aims to develop mobile interactive application software for translation of American Sign Language to speech and text and vice versa, to assist communication between hearing and speech impaired people with normal people. The language translator should be able to translate 26 alphabet gestures from the American Sign Language[1] into speech and text and vice versa.

We will basically acquire images using the inbuilt camera of the device and perform vision analysis, functions in the operating system and provide speech output through the inbuilt audio device.

Also to display the respective finger sign for a particular letter we will first acquire speech input using the inbuilt microphone of the device and then perform speech analysis functions in the operating system and provide visual sign output through the inbuilt display device.

There is a communication barrier existing between a normal person who is unaware of the sign language system and wishes to communicate with a deaf person. The objective of the project is to overcome these difficulties. The intended sign gestured by the user is to be translated into its corresponding alphabet using an interface which will ideally detect the accurate representation from its library of existing gestures and display it onscreen.

Furthermore, the proposed system can be improved and augmented to serve additional applications using technologies such as Virtual Reality, Artificial Intelligence, games and HCI (Human Computer Interaction)[2].
TABLE I

CONCLUSIONS DRAWN FROM RESEARCH

\begin{tabular}{|c|c|c|c|}
\hline $\begin{array}{l}\text { Sr. } \\
\text { No }\end{array}$ & $\begin{array}{c}\text { Paper } \\
\text { Authors } \\
\end{array}$ & Inferences & Drawbacks \\
\hline 1. & $\begin{array}{l}\text { Rung-Huei } \\
\text { Liang,Ming } \\
\text { Ouhyoung[1] }\end{array}$ & $\begin{array}{l}\text { The paper includes } \\
\text { grouping of } \\
\text { alphabets based on } \\
\text { gestures they } \\
\text { denote. } \\
\text { This makes gesture } \\
\text { recognition simpler. }\end{array}$ & $\begin{array}{l}\text { Data Glove is } \\
\text { used, which } \\
\text { makes the } \\
\text { system more } \\
\text { complex. }\end{array}$ \\
\hline 2. & $\begin{array}{l}\text { Sahib Singh, } \\
\text { Dr. Vijay } \\
\text { Kumar } \\
\text { Banga[2] }\end{array}$ & $\begin{array}{l}\text { This paper describes } \\
\text { a system that uses } \\
\text { red markers to } \\
\text { recognize the } \\
\text { gestures. } \\
\text { This increases the } \\
\text { accuracy rate of } \\
\text { recognition. }\end{array}$ & $\begin{array}{l}\text { The system is } \\
\text { limited to } \\
\text { minimal } \\
\text { gestures to } \\
\text { control PCs. } \\
\text { Red markers } \\
\text { may } \\
\text { inconvenience } \\
\text { the user. }\end{array}$ \\
\hline 3. & $\begin{array}{l}\text { Siddharth } \\
\text { Rautaray, } \\
\text { Anupam } \\
\text { Agarwal[3] }\end{array}$ & $\begin{array}{l}\text { This paper describes } \\
\text { a mechanism of } \\
\text { gesture recognition } \\
\text { using contours. } \\
\text { This eliminates the } \\
\text { need of external } \\
\text { devices and a } \\
\text { marker, simple } \\
\text { webcam is } \\
\text { sufficient. }\end{array}$ & $\begin{array}{l}\text { System is } \\
\text { limited to } \\
\text { minimal } \\
\text { gestures. }\end{array}$ \\
\hline
\end{tabular}

\section{Proposed System}

A. Image capturing through webcam

User is made to generate gestures in front of the camera, using solely a webcam as described in the paper by Rautauray and Agarwal[2] and relayed to the program for further processing. The camera is supposed to be fixed, and illumination slowly varying. Real-time constraints are being imposed for a careful design of the processing system. 


\section{B. Segmentation}

Separation of external and unnecessary factors from the image captured forms the crux of this section. Any sort of background disturbance or components of the image not required for processing are to be separated from the image of the gesture.

The unnecessary information is first removed. In particular, a background suppression procedure has been performed in the HSV colour space, in which the scene can be modelled discarding illumination variations. Thus focusing the attention on areas corresponding to human skin colour.

\section{Translation Process}

1) Location of finger or sign gesture by user gesture movement:

To overcome the hassle related to hardware sensors in the Data glove technology as proposed by Liang and Ouhyoung[1], we use the image generated by the webcam.

Once the image is stripped from the background and other inconsequential matter; the contours in the gesture are measured according to the shape formed by the hand [3]. The database constructed contains all the approved and accepted gestures by the ASL convention.

The contour deduced from the image is matched to the relevant sign in the database.

2) Initiating call and terminating call set up functions in Image processing using MATLAB:

This interface will be used to link the image acquisition process to the image processing process.

The journey from acquiring the image from webcam to accurately detecting the gesture intended by the user is controlled by the MATLAB skeleton.

3) Building of the GUI :

For the second aspect of the communication, a GUI (Graphical User Interface) is integral to the process of interaction between a disabled person and a normal layman.

While the detection of sign from a gesture is fairly free of GUI needs, the communication intended the other way round requires a presence of a GUI so that a normal person can relay his message to a disabled person without being inconvenienced.

4) Integrating all modules into GUI :

All the MATLAB frameworks, database skeleton, classes and functions need to be cohesively compacted into a structure that can function as an independent unit.

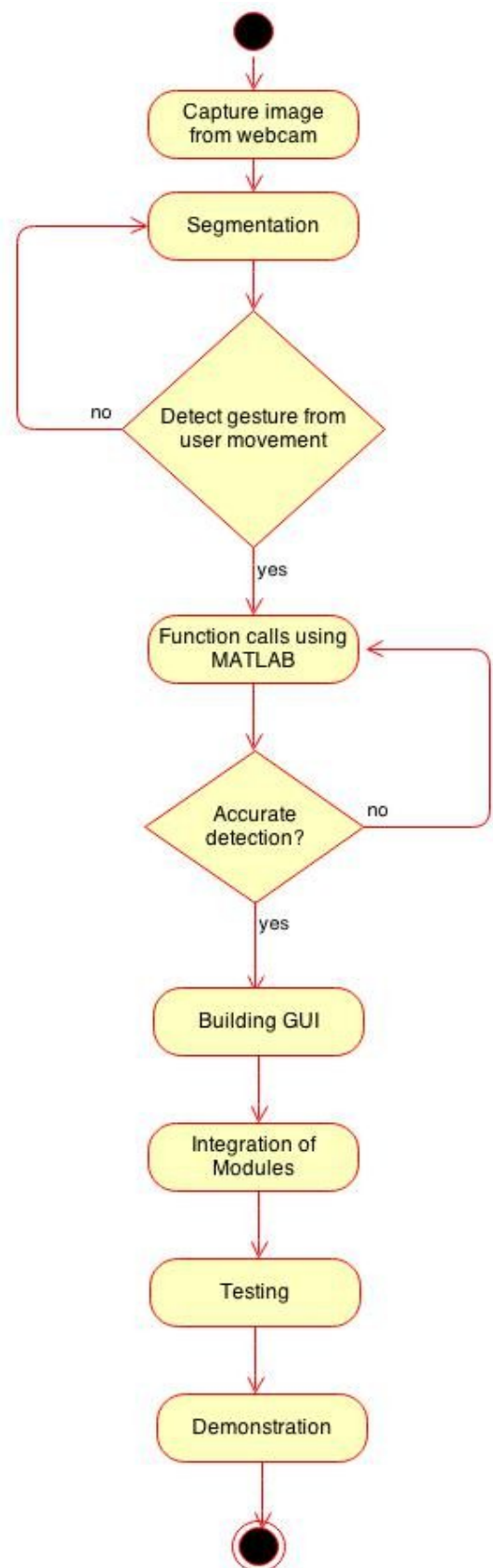

Fig. 1 Activity Diagram for Translation Process

5) Testing:

This process involves evaluating whether the finished product adheres to the requirements specified in the documentation of the project. Functional and structural entities will be judged for their efficiency. Accuracy of identification of gesture, conversion from sign to text and speech, dynamicity of the database, translation from text to sign, addition of customised signs and ease of usage are some of the factors intended to be tested. In order to test the proposed gesture recognition system, analysis parameters such as robustness, scalability, computational efficiency and user's tolerance can be considered[2]. 
6) Demonstration:

The prototype of the finished model will be demonstrated in front of the panel of examiners to be evaluated and graded.

\section{III.Image Processing and Feature Extraction}

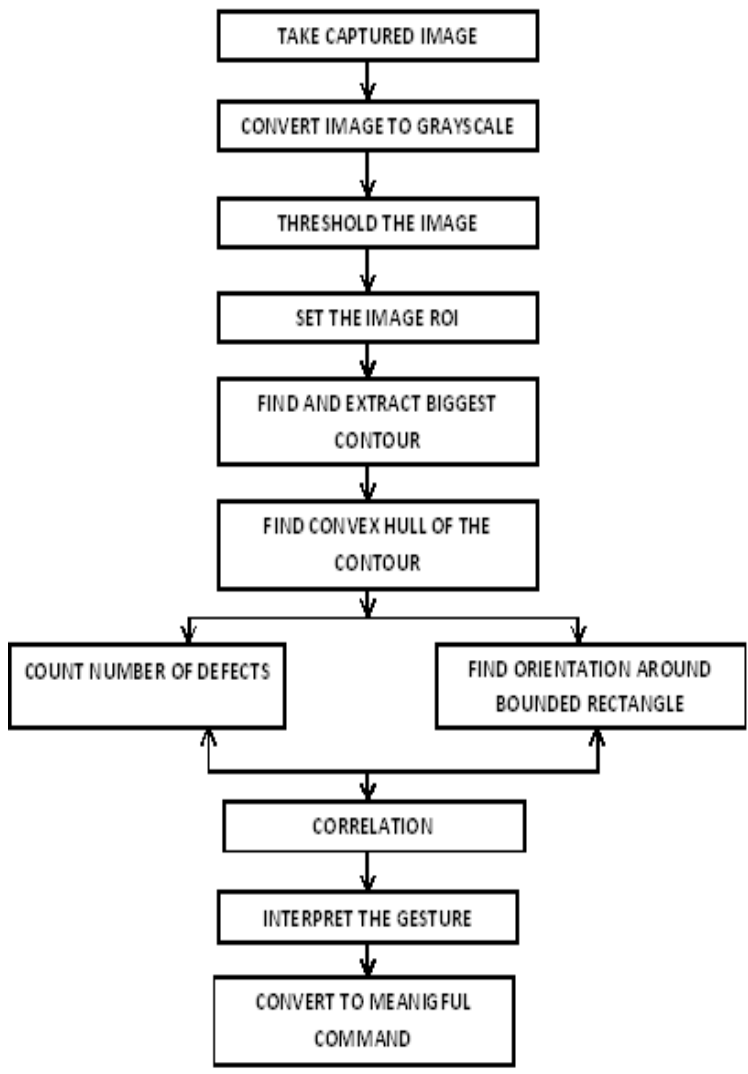

Fig.2 Image Pre-processing and Feature Extraction procedure

\section{A. Steps for hand detection}

1. Read the image

2. Convert to gray scale[4]

3 . In the sample image, the background illumination is brighter in the centre of the image than at the bottom. As a pre-processing step before analysis, make the background uniform and then convert the image into a binary image. To make the background illumination more uniform, create an approximation of the background as a separate image and then subtract this approximation from the original image.

4. As a first step to creating a background approximation image, remove the entire foreground using morphological opening.

5. The opening operation has the effect of removing objects that cannot completely contain the structuring element

6. Subtract the background approximation image, from the original image and view the resulting image[2].

7. Use imadjust to increase the contrast of the processed image.
8. Create a binary version of the processed image[6] using thresholding[2][6]. The binary conversion can be done using the method of image segmentation [5]

The background pixels can be converted into black color pixels and pixels containing our area of interest can be converted into white colour pixels. In this way preprocessing procedure is carried out.

\section{B. Reference Database Design}

The database contains images, their corresponding IDs, and text meanings of the sign. To this end, Microsoft Access was used to build it. This is because, in addition to its ease of use, it provides us with the ability to store images and sounds - which is the main purpose of the database.

We used an ODBC data source to connect the database to our $\mathrm{C}$ code. Our $\mathrm{C}$ code reads-in from the text file that was produced by the gesture recognition module the ID of the image that yielded a successful match. A select SQL statement is used to locate the image in the database in order to get the text meaning of the sign. The corresponding sound is generated after the text-to-sound

\section{Additions to Existing Database and Text to Sound Converter}

In order to make the proposed system dynamic, the user will be provided with the option of inserting new signs to the database. For further simplification sound has been added to the database and also the feature of inserting meaning for a new sign as a string of text to the database. This text is then processed using our text-to-sound module or a Sound Blaster Card[1] to produce a wave file of the corresponding sound[6]. This sound file is added to the sound files folder and thus the proposed system can now comprehend a new sign.

\section{IV.CONCLUSION}

This project is a small step towards helping a physically challenged people and lot more can be done to make the product more sophisticated, user friendly \&efficient. Using more memory and powerful microprocessor, more languages can be covered. This project can be modified to make it compatible with mobile phones. We can increase the range of product by using more powerful trans-receiver module.

\section{ACKNOWLEDGMENT}

We express our gratitude to our project guide Prof Sumita Chandak, who provided us with all the guidance and encouragement and making the lab available to us at any time. We also would like to deeply express our sincere gratitude to Project coordinators.

We are eager and glad to express our gratitude to the Head of the Information Technology Dept. Prof Neelima Pathak ,for her approval of this project. We are also thankful to her for providing us the needed assistance, detailed suggestions and also encouragement to do the project. converter reads from a text-file. 
We would like to deeply express our sincere gratitude to our respected principal Prof. Dr. Shrikant Kallurkar and the management of Atharva College of Engineering for providing such an ideal atmosphere to build up this project with well-equipped library with all the utmost necessary reference materials and up to date IT Laboratories.

We are extremely thankful to all staff and the management of the college for providing us all the facilities and resources required.

\section{REFERENCES}

[1] Rung-Huei Liang, Ming Ouhyoung," A Real-time Continuous Alphabetic Sign Language to Speech Conversion VR System," Communications \& Multimedia Lab., Computer Science and Information Engineering Dept., National Taiwan University, Taipei, Taiwan.

[2] Siddharth S. Rautaray, Anupam Agrawal," Real time hand gesture recognition system for dynamic applications," International Journal of UbiComp (IJU), Indian Institute of Information Technology Allahabad, India, Vol.3, No.1, January 2012.

[3] Sahib Singh1,Dr. Vijay Kumar Banga,"Gesture control algorithm for personal computers," ISSN: 2319 - 1163,Volume: 2 Issue: 5, Department of Electronics and Communication Engineering,Punjab, India.

[4] N.Hema,Ms. P. Thamarai ,Dr. T.V.U. Kiran Kumar,"HandiCom: Handheld Deaf and Dumb Communication Device based on Gesture to Voice and Speech to Image/Word Translation with SMS Sending and Language Teaching Ability, "IJESRT,ISSN: 2277 9655,Department of Electronics and Communication Engineering, Tamil Nadu, India.

[5] AditiKalsh,N.S. Garewal,"Sign Language Recognition for Deaf \& Dumb, "IJARCSSE, Department of Electronics \& Communication, GNE/ PTU, India, Volume 3, Issue 9, September 2013.

[6] Sawant Pramada1 ,Deshpande Saylee, Nale Pranita, Nerkar Samiksha, Mrs. Archana S. Vaidya, "Intelligent Sign Language Recognition Using Image Processing", GES's R. H. Sapat College

[7] of Engineering, Nashik, India IOSR Journal of Engineering (IOSRJEN),e-ISSN: 2250-3021, p-ISSN: 2278-8719 Vol. 3, Issue 2,||V2|| PP 45-51 ,Feb. 2013

\section{BIOGRAPHIES}

Neha Poddar is currently pursuing B.E in Information Technology from Atharva College of Engineering, Mumbai, India. Her research area includes .NET, Matlab and Java.

Shrushti Rao is currently pursuing B.E in Information Technology from Atharva College of Engineering, Mumbai, India. Her research area includes .NET, SQL and Security Systems.

Shruti Sawant is currently pursuing B.E in Information Technology from Atharva College of Engineering, Mumbai, India. Her research area includes Network Studies, HTML, Web Designing and Java.

Vrushali Somavanshi is currently pursuing B.E in Information Technology from Atharva College of Engineering, Mumbai, India. Her research area includes HTML, DBMS and UI/UX.

Sumita Chandak is currently working as Assistant Professor in the department of Information Technology at Atharva College of Engineering, Mumbai, India. Her research area includes Image/Video Processing and Networking. 